\title{
Melanoma da Coroideia
}

\section{Choroidal Melanoma}

Inês COUTINHO ${ }^{1}$, Tânia TEIXEIRA², Paulo César SIMÕES ${ }^{3,4}$, João Casalta LOPES², Margarida BORREGO², Júlia FERNANDES ${ }^{5}$, João CABRAL ${ }^{6}$, Isabel PRIETO ${ }^{1}$, Rui PROENÇA ${ }^{5}$

Acta Med Port 2017 Jul-Aug;30(7-8):573-577 - https://doi.org/10.20344/amp.8535

\section{RESUMO}

O melanoma da coroideia é o tumor primário intraocular maligno mais frequente em adultos. Nenhum dos diferentes tratamentos disponíveis oferece vantagens de sobrevida recorrendo-se, cada vez mais, a tratamentos conservadores como a braquiterapia, a qual passou a estar disponível em Portugal desde 2013. Neste artigo revemos as características clínicas, factores de risco, diagnóstico, exames complementares e opções terapêuticas no melanoma da coroideia.

Palavras-chave: Melanoma; Neoplasias da Coroideia

\section{ABSTRACT}

Choroidal melanoma is the most common primary intraocular malignant tumor in adults. None of the different treatments available offers advantages of survival, resorting more and more to conservative treatments such as brachytherapy, which has been available in Portugal since 2013. In this article we review the clinical characteristics, risk factors, diagnosis, complementary exams and therapeutic options in choroidal melanoma.

Keywords: Choroid Neoplasms; Melanoma

\section{INTRODUÇÃO}

O melanoma primário pode afetar diversas estruturas oculares, desde a pálpebra, conjuntiva, úvea (coroideia, corpo ciliar e íris) e muito raramente a órbita, sendo responsável por cerca de $3 \%-5 \%$ de todos os melanomas. ${ }^{1-7}$

É importante referir que o melanoma intraocular (também designado melanoma da úvea) é considerado uma identidade diferente do melanoma cutâneo e da conjuntiva. $\mathrm{O}$ primeiro tem origem nos melanócitos da mesoderme e metastiza por via hemática, em contraste com o tumor da pele e mucosas que tem origem na ectoderme e metastiza, preferencialmente, por via linfática. ${ }^{4}$

É sobre o melanoma primário da coroideia, que corresponde a $85 \%$ dos melanomas oculares, que incide este trabalho de revisão. Em comparação com o melanoma da íris, apresenta pior prognóstico e melhor que o melanoma do corpo ciliar. ${ }^{1,4,6}$

\section{Epidemiologia e fatores de risco}

O melanoma da coroideia é o tumor intraocular maligno primário mais comum no adulto, e o segundo tipo de melanoma primário mais frequente, imediatamente após o melanoma cutâneo. Estima-se uma incidência anual de aproximadamente 4 - 10 casos por 1 milhão de habitantes. $1,2,3,6,8-10$

É mais frequente na sexta década de vida e, ao contrário do que se observa na maioria das neoplasias, a sua incidência parece decrescer em idades mais avançadas. Distribui-se igualmente em ambos os sexos, com predile- ção por indivíduos de pele e olhos claros. . $^{1,4,7,11}$

Acredita-se que a maioria dos melanomas da coroideia se desenvolva a partir de nevus pré-existentes, embora o crescimento de novo também possa ocorrer.

Outros factores de risco são a melanocitose congénita ocular e óculo-dérmica, síndrome de nevus displásico e neurofibromatose tipo 1. Não há evidência científica consistente quanto ao papel da radiação ultravioleta, ao contrário do que ocorre nos casos de melanoma da pele e mucosas. $4,5,7,8,11-13$

\section{Apresentação clínica}

As manifestações clínicas são inespecíficas e não contribuem para o diagnóstico, sendo muitas vezes um achado acidental num exame oftalmológico de rotina.

Numa fase inicial os doentes são assintomáticos, mas quando o tumor atinge grandes dimensões ou se localiza próximo ao nervo óptico ou mácula, pode causar queixas de baixa visão, miodesópsias e fotópsias. A dor é rara e um sintoma sugestivo de mau prognóstico.4,5,7,10,11,13

Ao exame oftalmológico, o melanoma da coroideia apresenta-se como uma lesão elevada subretiniana, geralmente única e unilateral, com uma coloração que varia de amelanocítica (15\% - 20\%) a pigmentada castanho-acinzentada, sendo raramente preto escuro. Tem uma conformação nodular (75\%) (Fig. 1), em cogumelo (20\%) (Fig. 2) ou difusa (5\%). Subjacente ao tumor é possível observar alterações do epitélio pigmentado da retina como atrofia,

\footnotetext{
1. Serviço de Oftalmologia. Hospital Fernando Fonseca. Amadora. Portugal.

2. Serviço de Radioncologia. Centro Hospitalar e Universitário de Coimbra. Coimbra. Portugal.

3. Serviço de Radioterapia. Centro Hospitalar e Universitário de Coimbra. Coimbra. Portugal.

4. Unidade de Biofísica. Faculdade de Medicina. Universidade de Coimbra. Coimbra. Portugal.

5. Serviço de Oftalmologia. Centro Hospitalar e Universitário de Coimbra. Coimbra. Portugal.

6. Serviço de Oftalmologia. Hospital da Luz. Lisboa. Portugal.

$\triangle$ Autor correspondente: Inês Coutinho. inescorga@gmail.com

Recebido: 03 de dezembro de 2016 - Aceite: 08 de junho de 2017 | Copyright @ Ordem dos Médicos 2017
} 


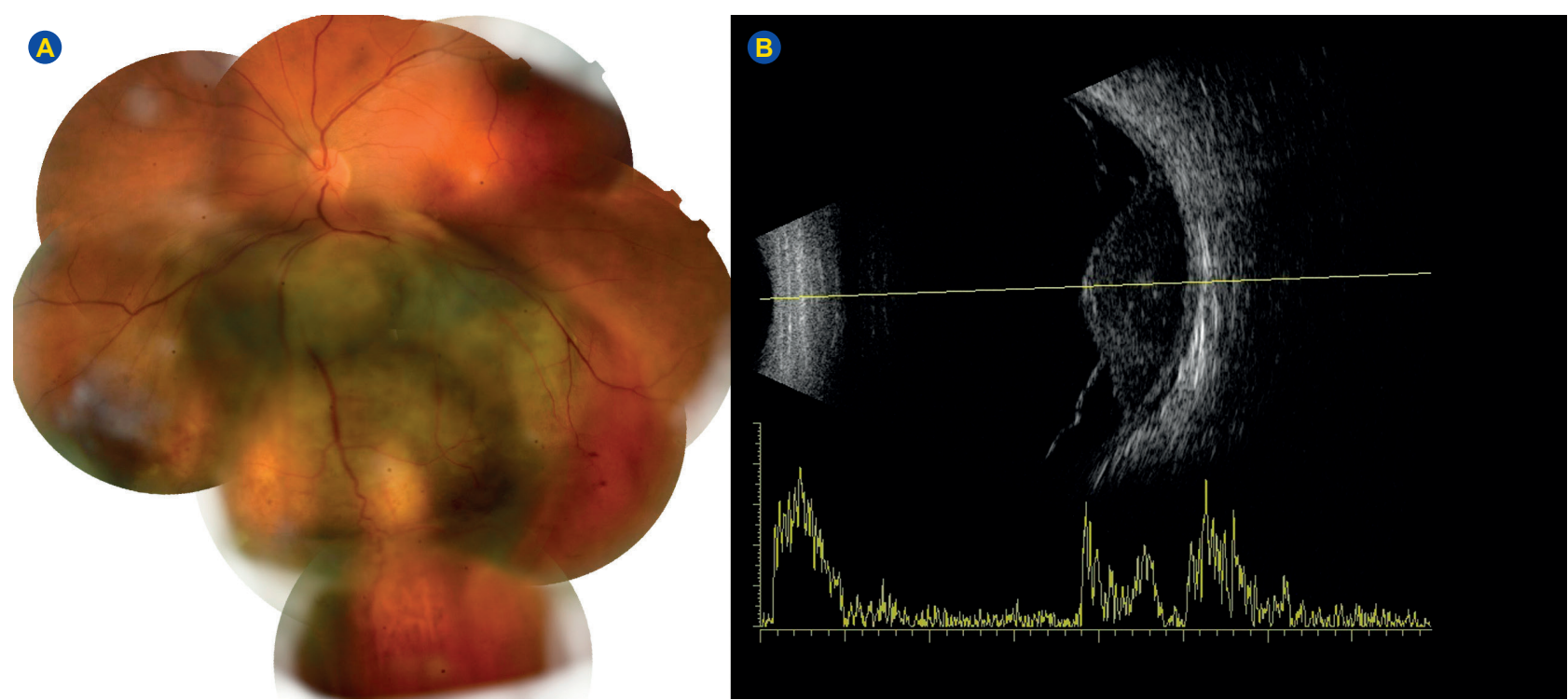

Figura 1 - A: Retinografia; B: Ecografia ocular modo A e B. Melanoma da coroideia nodular ou dome-shaped, em que a membrana de Bruch está integra

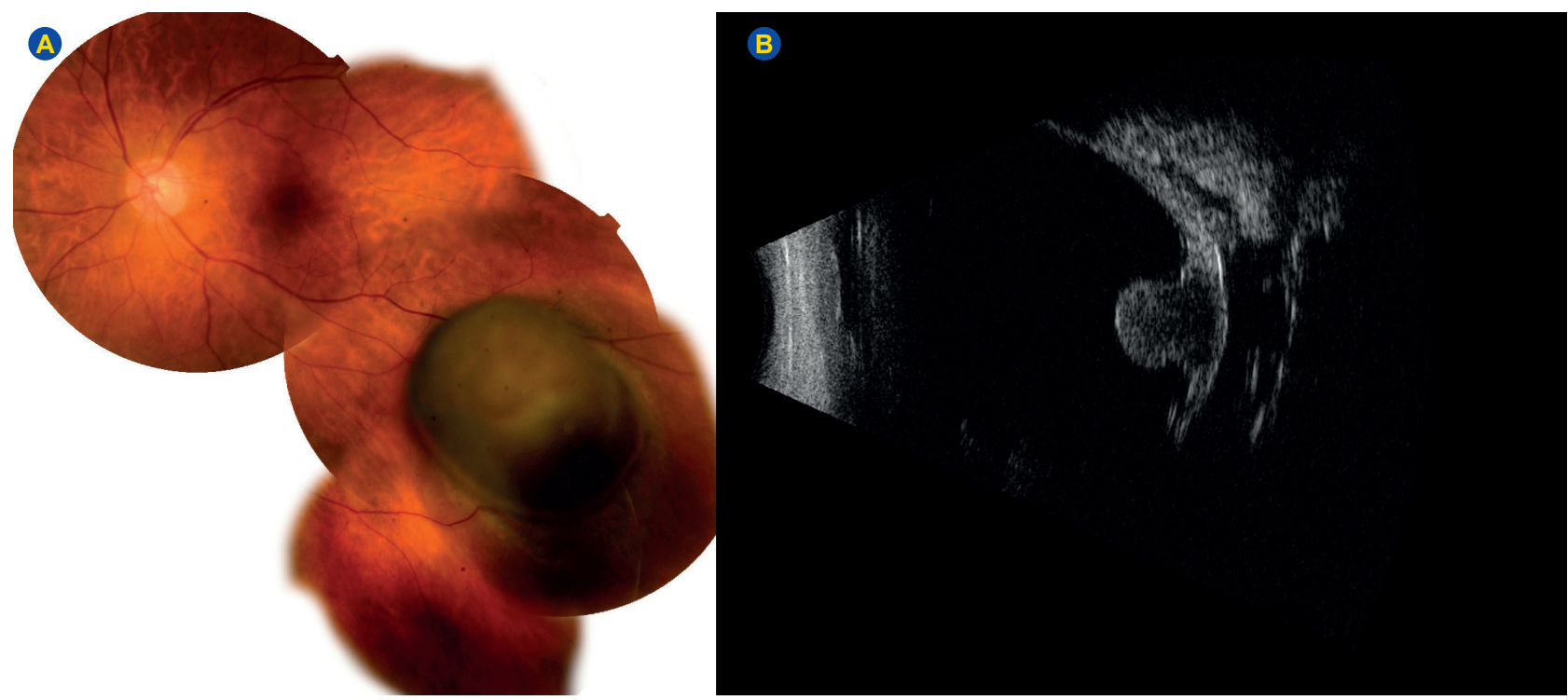

Figura 2 - A: Retinografia; B: Ecografia ocular modo B. Melanoma em forma de cogumelo, mushroom ou colar button shape, em que há rotura da membrana de Bruch, sendo esta apresentação muito sugestiva do diagnóstico de melanoma da coroideia

pigmentação alaranjada por acúmulo de lipofuscina ou descolamento exsudativo da retina..$^{5,7,10,12,13}$

\section{Diagnóstico}

O diagnóstico de melanoma da coroideia é, na grande maioria dos casos, preciso, tendo o estudo Collaborative Ocular Melanoma Study (COMS) relatado uma taxa de erro no diagnóstico de $0,48 \%$ e concluindo que o maior desafio do melanoma da úvea não é o seu correto diagnóstico mas antes a determinação do melhor tratamento. ${ }^{2,14}$
A fundoscopia sob midríase é essencial, sendo a ecografia ocular (modo A e B), com ou sem doppler, o melhor exame complementar de diagnóstico para o melanoma da coroideia.

A ecografia de modo B permite avaliar a forma e tamanho tumoral (Tabela 1), planear a intervenção terapêutica mais indicada e realizar o seguimento dos doentes após tratamento. No modo A, o tumor apresenta caracteristicamente refletividade interna média a baixa (Fig. 3). Este padrão de refletividade auxilia no diagnóstico diferencial,

Tabela 1 - Definição pelo tamanho do melanoma da coroideia segundo o estudo COMS

\begin{tabular}{lcc}
\hline Tamanho & Pequeno & Médio \\
\hline Espessura apical & $1,5-2,4 \mathrm{~mm}$ & $2,5-10 \mathrm{~mm}$ \\
Diâmetro & $5-16 \mathrm{~mm}$ & $<16 \mathrm{~mm}$ \\
\hline
\end{tabular}




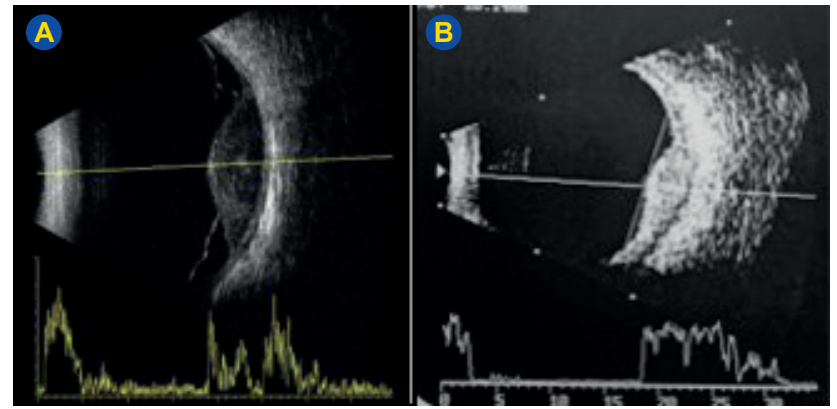

Figura 3 - A: Aspeto ecográfico de melanoma da coroideia. Ecografia de modo A revela pico de maior refletividade no ápice da lesão correspondendo à retina, seguida de uma reflectividade interna da lesão media a baixa e com redução gradual dos ecos em direcção a base (ângulo k), seguida por um último pico que corresponde a esclerótica; B: Aspeto ecográfico de hemangioma da coroideia

uma vez que as metástases e os nevus da coroideia apresentam refletividade moderada a elevada e o hemangioma da coroideia um pico inicial elevado mantendo alta refletividade. ${ }^{1,4,7,10,15}$

A fundoscopia aliada à ecografia permite estabelecer o diagnóstico com confiança em mais de $95 \%$ dos casos, sendo muito rara a necessidade de efetuar uma biópsia, procedimento este de difícil realização técnica.

Outros exames disponíveis que podem auxiliar no diagnóstico em casos atípicos ou com maiores dúvidas diagnósticas são a angiografia fluoresceínica e com verde de indocianina, tomografia de coerência óptica (OCT), tomografia computorizada (TC) e ressonância magnética (RM) de órbitas. ${ }^{1,4,7,10,15}$

$\mathrm{Na} \mathrm{RM}$, a melanina é hiperintensa em T1 e hipointensa em T2, contudo este padrão típico não está presente nos tumores amelanocíticos e não é patognomónico dos melanomas da coroideia, confundindo-se frequentemente com o sinal semelhante da hemossiderina de uma hemorragia antiga. $^{7}$

\section{Diagnóstico diferencial}

O diagnóstico diferencial de melanoma da coroideia faz-se tanto com patologias benignas como malignas. Entre as principais encontram-se o nevus da coroideia, hemangioma da coroideia, melanocitoma, metástases da coroideia, hiperplasia congénita do epitélio pigmentado da retina e hematoma supracoroideu (Fig. 4). ${ }^{10,13,15}$

Em casos de pequenos melanomas, o diagnóstico diferencial com nevus da coroideia pode ser complexo. O nevus da coroideia é, geralmente, assintomático, plano ou com menos de $1 \mathrm{~mm}$ de espessura e base menor que 5 $\mathrm{mm}$, com drusas. ${ }^{7}$

Os fatores de risco relacionados com a transformação maligna do nevus da coroideia são: espessura maior que 2 $\mathrm{mm}$, presença de líquido sub-retiniano, sintomas, presença de pigmento laranja (lipofuscina), localização $<3 \mathrm{~mm}$ do disco óptico, vazio acústico na ecografia e ausência de drusa ou halo hipopigmentado ao redor da lesão.

Shields formulou a mnemónica TFSOM (To Find Small
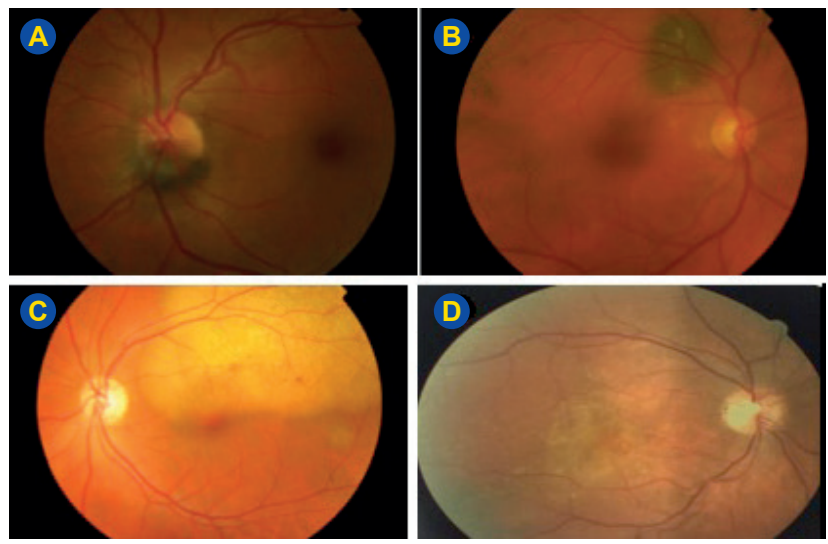

Figura 4 - A: Melanocitoma; B: Nevus da coroideia; C: Metástase da coroideia; D: Hemangioma da coroideia

Ocular Melanoma) - Thickness (T), Subretinal Fluid (F), Symptoms (S), Orange pigment (O) e margin touching optic $\operatorname{disc}(\mathrm{M}) .^{5}$

O melanocitoma é uma variante do nevus melanocítico, fortemente pigmentado, com uma coloração negra escura e localizado preferencialmente na região peripapilar.

As metástases coroideias apresentam-se como lesões geralmente amelanocíticas e podem ser bilaterais e múltiplas, localizando-se frequentemente no pólo posterior e com características ecográficas distintas. ${ }^{15,18}$ Se a suspeita for de metástase coroideia, deve-se tentar identificar o tumor primário, já que em $30 \%$ - 40\% dos doentes não existe história oncológica quando apresentam a metástase ocular. Os tumores que frequentemente metastizam para o olho são o da mama e do pulmão. ${ }^{16,17}$

O hemangioma isolado da coroideia é um tumor vascular benigno de coloração alaranjada, em forma de placa discretamente elevada, localizada frequentemente no pólo posterior. A complicação mais comum é o descolamento exsudativo da retina. A ecografia e a angiografia com verde de indocianina auxiliam no seu diagnóstico. ${ }^{15,18}$

\section{Estadiamento}

No momento do diagnóstico, deve ter-se presente que o principal local de metastização é o fígado (90\%), seguindo-se o pulmão e restantes órgãos. ${ }^{4,6}$

Desta forma, na avaliação inicial, os doentes realizam hemograma e provas hepáticas (AST; ALT; GGT, FA e eventualmente $\mathrm{LDH}$ e CEA), ecografia ou TC abdominal e radiografia do tórax.

Um diagnóstico e tratamento precoces permitem conservar o olho e, em alguns casos, alguma acuidade visual, bem como eventualmente diminuir a ocorrência de metástases. Alguns estudos demonstraram que o melanoma da coroideia pode ser considerado uma doença sistémica, em que no momento do diagnóstico pode já existir células neoplásicas em circulação no sangue periférico, sem serem detetadas clinicamente (micrometástases). Contudo, só alguns doentes irão desenvolver doença metastática, por fatores ainda não totalmente conhecidos, a qual pode 
ocorrer até décadas após o diagnóstico do tumor primário e que está associada à mortalidade destes tumores.

\section{Tratamento}

Nas últimas décadas, o tratamento do melanoma da coroideia sofreu grandes avanços, recorrendo-se, cada vez, mais a tratamentos conservadores em vez da enucleação.

A hipótese de que a enucleação podia ser um fator promotor de disseminação de células malignas para a corrente sanguínea e tecidos orbitários (hipótese de Zimmerman-McLean-Foster), aliada a outros estudos que defendiam que as células tumorais produzem proteínas anti-angiogénicas, e que a remoção do tumor primário faz perder este equilíbrio, predispondo ao crescimento de micrometástases, impulsionou o desenvolvimento de outras modalidades terapêuticas. ${ }^{19,20}$

O estudo Collaborative Ocular Melanoma Study (COMS) ao demonstrar que a enucleação, associada ou não a radioterapia externa, não apresenta benefício em termos de sobrevida, nem na prevenção de metástases, quando comparada à braquiterapia em melanomas de tamanho médio, levou a uma preferência atual por terapêuticas conservadoras, que permitem conservar o globo ocular, e em alguns casos a visão. Em estudos recentes, a radioterapia consegue um controlo local e sobrevivência comparável com a enucleação. ${ }^{2,14,19}$

No entanto, e apesar do avanço do conhecimento médico, ainda não está determinado qual a melhor terapêutica, sendo a enucleação, radioterapia, ressecção local, fotocoagulação e a termoterapia os métodos atualmente disponíveis.

A escolha por uma terapêutica a instituir recai no estudo do tamanho tumoral, envolvimento extraocular, presença de metástases, idade e estado geral do doente, assim como acuidade visual do olho adelfo. 2,7,16,19,20

Atualmente, a enucleação é reservada para tumores grandes, mais de $10 \mathrm{~mm}$ de espessura e $16 \mathrm{~mm}$ de base, que contraindiquem um tratamento conservador.

Para tumores pequenos, até $2 \mathrm{~mm}$ de espessura e sem crescimento tumoral documentado, é apenas indicado a vigilância periódica.

A radioterapia é a primeira escolha nos tumores até 10 $\mathrm{mm}$ de espessura e $16 \mathrm{~mm}$ de base. Está contraindicada em tumores com mais de $15 \mathrm{~mm}$ de espessura e com extensão extra-escleral superior a $5 \mathrm{~mm}$ de espessura. Para extensões extra-escleral superiores, a exenteração orbitária, seguida ou não de radioterapia externa adjuvante, pode ter indicação.

A quimioterapia sistémica é o tratamento que se oferece aos doentes com metástases à distância no momento do diagnóstico, sendo atualmente apenas um tratamento paliativo.

Em Portugal, foi inaugurado em 2013 no Centro Hospitalar e Universitário de Coimbra, o Centro de Referência para Tratamento dos Tumores Oculares, que dispõe de braquiterapia episcleral para o tratamento de melanomas da úvea.

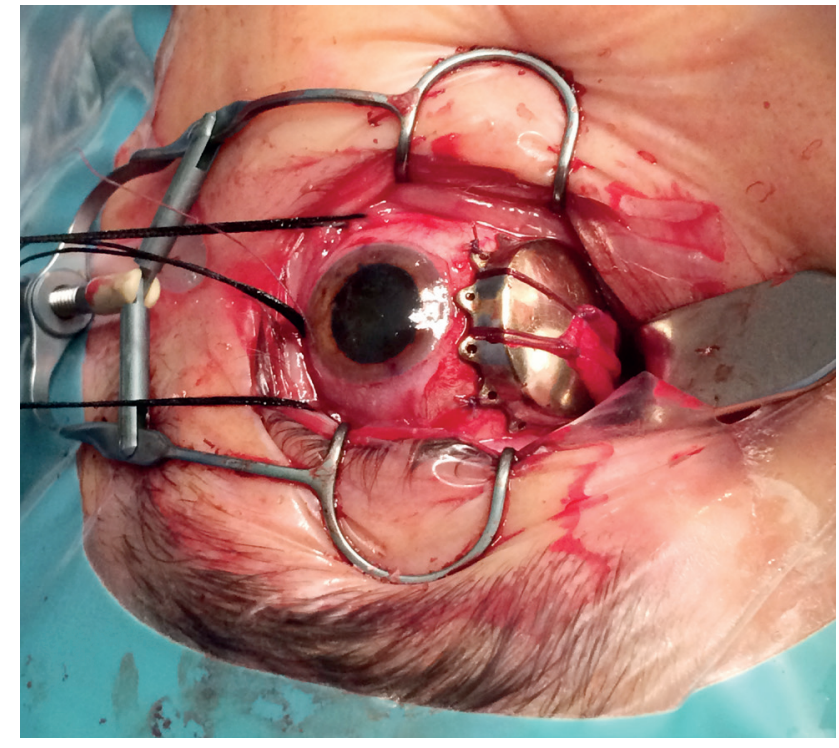

Figura 5 - Colocação intra-operatória de uma placa episcleral com sementes de lodo ${ }^{125}$

A braquiterapia é uma forma de radioterapia na qual uma placa carregada com fontes radioativas é suturada na esclerótica adjacente ao tumor intraocular (Fig. 5), permanecendo implantada por um período de tempo determinado pelo estudo dosimétrico. O tipo de placa, o número de sementes a colocar e a sua distribuição irão depender do tamanho do tumor e da sua localização (existem placas com entalhe para tumores perto do nervo óptico), de forma a que a isodose de $85 \mathrm{~Gy}$ possa envolver toda a lesão com margem de segurança. A placa é colocada e retirada sob anestesia geral, por uma equipa multidisciplinar composta por oftalmologistas, radio-oncologistas e físicos médicos.

Durante o período de tratamento, o doente fica internado em ambiente controlado e com visitas condicionadas. O quarto de internamento está preparado com um biombo protetor de radiações e o doente dispõe de óculos com vidro plumbíneo que coloca sempre que está acompanhado, para proteção das visitas. Não são necessárias outras medidas especiais de proteção, e nenhum material ou resíduos ficam contaminados com radiação após a remoção da placa terapêutica.

Vários isótopos podem ser usados nas placas de braquiterapia, sendo o lodo $^{125} \mathrm{o}$ de eleição mundial e o usado em Portugal, pelo seu melhor perfil de segurança, pela versatilidade, e reutilização.

Os estudos mostram que a braquiterapia é eficaz, sendo a taxa de insucesso e necessidade de enucleação de $10 \%$ e $13 \%$ em cinco anos, respectivamente..$^{10,16,21}$

Está associada a complicações como retinopatia da radiação, neuropatia óptica e catarata que poderão ocorrer em cerca de $50 \%$ dos doentes após dois anos, comprometendo a acuidade visual..$^{10,16,19}$

A braquiterapia tem indicações e taxas de sucesso relativamente semelhantes às da irradiação com feixe externo de protões, outra técnica de radioterapia, contudo menos difundida mundialmente, pois necessita de equipamentos e instalações complexas. 
Em relação às complicações, de um modo geral a radioterapia por protões induz mais complicações no segmento anterior do olho, enquanto na braquiterapia é o segmento posterior que tem maior risco de complicações.

\section{Seguimento e prognóstico}

Os doentes com melanoma ocular devem ser acompanhados por oftalmologistas especializados nesta área.

Sabe-se que nos primeiros anos existe uma maior probabilidade de desenvolvimento de metástases, pelo que, durante este período a vigilância é mais apertada. Assim, no primeiro ano a avaliação clínica, analítica e imagiológica com ecografia hepática e ocular é realizada a cada três meses, sendo nos anos seguintes e até completar cinco anos semestral e depois com periodicidade anual.

Genericamente o melanoma da úvea é uma doença grave com prognóstico reservado.

O prognóstico depende da idade à data do diagnósti- co, dimensão e localização do tumor, histologia (tipo epitelióide, infiltrado linfocitário e alto índice mitótico), extensão extra-escleral, fatores citogenéticos (monossomia do cromossoma 3 e ampliação do braço longo do cromossoma 8), entre outros fatores ainda em estudo. 1,7,8,11,13,19,21

Estima-se que a taxa de mortalidade do melanoma da coroideia aos 10 anos seja de $50 \%$, mas na presença de doença metastática este costuma ser fatal em média após 6 a 12 meses. $6,8,9,16,21$

\section{CONFLITOS DE INTERESSE}

Os autores declaram não terem qualquer conflito de interesse relativamente ao presente artigo.

\section{FONTES DE FINANCIAMENTO}

Os autores declaram não terem beneficiado de qualquer financiamento

\section{REFERÊNCIAS}

1. Singh AD, Bergman L, Seregard S. Uveal melanoma: epidemiologic aspects. Ophthalmol Clin North Am. 2005;18:75-84.

2. Hawkins BS, Collaborative Ocular Melanoma Study Group. The Collaborative Ocular Melanoma Study (COMS) randomized trial of pre-enucleation radiation of large choroidal melanoma: IV. Ten-year mortality findings and prognostic factors. COMS report number 24 . Am J Ophthalmol. 2004;138:936-51.

3. Singh AD, Turell ME, Topham AK .Uveal melanoma: trends in incidence, treatment, and survival. Ophthalmology. 2011;118:1881-5.

4. Yonekawa Y, Kim IK. Epidemiology and management of uveal melanoma. Hematol Oncol Clin North Am. 2012;26:1169-84.

5. Shields $\mathrm{CL}$, Shields JA. Ocular melanoma: relatively rare but requiring respect. Clin Dermatol. 2009;27:122-33.

6. Kaliki S, Shields CL, Shields JA. Uveal melanoma: Estimating prognosis. Indian J Ophthalmol. 2015;63:93-102.

7. Laranjeira J, Cabral J, Silva J, Luis A. Tumores da úvea. Oftalmologia Clínica. 2016:426-37.

8. Proença R. Factores de prognóstico do melanoma da úvea. Experientia Ophthalmologica. Coimbra. 1999; vol 25; n¹.

9. McCannel TA. Fine-needle aspiration biopsy in the management of choroidal melanoma. Curr Opin Ophthalmol. 2013:24:262-6.

10. Garcia-Valenzuela E. Choroidal Melanoma. Medscape. 2016. [consultado 2016 out 14]. Disponível em: http://emedicine.medscape. com/article/1190564-overview.

11. Augsburger JJ, Corrêa ZM, Trichopoulos N. An alternative hypothesis for observed mortality rates due to metastasis after treatment of choroidal melanomas of different sizes. Trans Am Ophthalmol Soc. 2007;105:54-

9.

12. Neto R, Andrade G. Melanoma de coróide. In: Atlas de Doenças da Mácula. Lima LH, Andrade GC, Maia A, Rodrigues EB, Farah ME, editores. $1^{\mathrm{a}}$ ed. Rio de Janeiro: Elsevier; 2015. p.438-48.

13. Shields CL, Manalac J, Das C, Ferguson K, Shields JA. Choroidal melanoma: clinical features, classification, and top 10 pseudomelanomas. Curr Opin Ophthalmol. 2014;25:177-85.

14. Choroidal Melanoma Update: Collaborative Ocular Melanoma Study (COMS) Results. Focal Points. American Academy of Ophthalmology. (section 1 of 3). June 2005.

15. Yanoff M, Duker JS. Ophthalmology. $4^{\text {th }}$ ed. London: Elsevier/Saunders; 2013.

16. Alves M. latrogenias e manifestações oculares de doenças sistémicas e oncologia ocular. In: Série Oftalmologia Brasileira. $3^{\mathrm{a}}$ ed. Rio de Janeiro: Gen - Guanabara Koogan; 2013.

17. Arepalli S, Kaliki S, Shields CL. Choroidal metastases: origin, features, and therapy. Indian J Ophthalmol. 2015;63:122-7.

18. Ophtalmic Pathology and Intraocular Tumors in Basic and Clinical Science Course, 2016-2017. San Francisco: American Academy of Ophthalmology; 2016.

19. Damato B. Does ocular treatment of uveal melanoma influence survival? Br J Cancer. 2010;103:285-90

20. Singh AD, Rennie IG, Kivela T, Seregard S, Grossniklaus H. The Zimmerman-McLean-Foster hypothesis: 25 years later. Brit $\mathrm{J}$ Ophthalmol. 2004;8:962-7.

21. Singh AD, Seregard S. Esaso Course Series. In: Ocular tumors. Karger; 2016. 